\title{
Oculometric Behavior Assessed by Pupil Response is Altered in Adolescent Depression
}

\author{
N. SEKANINOVA ${ }^{1,2}$, I. ONDREJKA $^{3}$, L. BONA OLEXOVA ${ }^{1,2}$, Z. VISNOVCOVA $^{1,2}$, \\ M. MESTANIK ${ }^{1,2}$, D. CESNEKOVA ${ }^{3}$, A. HAMRAKOVA ${ }^{1,3}$, I. TONHAJZEROVA $^{1,2}$
}

${ }^{1}$ Department of Physiology, Jessenius Faculty of Medicine in Martin, Comenius University in Bratislava, Martin, Slovak Republic, ${ }^{2}$ Biomedical Center Martin, Jessenius Faculty of Medicine in Martin, Comenius University in Bratislava, Martin, Slovak Republic, ${ }^{3}$ Psychiatric Clinic, Jessenius Faculty of Medicine in Martin, Comenius University in Bratislava, University Hospital Martin, Martin, Slovak Republic

Received March 21, 2019

Accepted October 21, 2019

\section{Summary}

Oculometric behaviour assessed by pupil response provides important information about central autonomic activity and emotional regulation. However, studies regarding pupil response to emotional stimuli in adolescent depression are rare. We aimed to study emotional-linked pupil response in adolescent depression. Twenty depressive adolescents (average age: $15.4 \pm 1.2$ years) and 20 age/gender-matched healthy subjects were examined. Average pupil diameter assessed by eye-tracking and pupillary light reflex were evaluated during protocol baseline, free-view task, recovery phase. Regarding right eye, significantly greater initial pupil diameter and pupil dilation to positive pictures free-viewing ( $p=0.013, p=0.031$, respectively), significantly slower average and maximum constriction velocity in baseline and positive pictures free-viewing $(p=0.036, p=0.050$, $p=0.021, p=0.015$, respectively), significantly slower maximum constriction velocity in recovery phase $(p=0.045)$, and significantly faster average dilation velocity in negative pictures free-viewing $(p=0.042)$ were observed in depressive group. Regarding left eye, significantly lower constriction percentual change in negative pictures free-viewing $(p=0.044)$ and significantly greater baseline pupil diameter and pupil diameter at the peak of constriction in positive vs. negative pictures freeviewing ( $p=0.002, p=0.015$, respectively) were observed in depressive group. Our study revealed discrete central autonomic dysregulation - parasympathetic hypoactivity associated with relative sympathetic dominance influenced by impairments in emotional processing already in adolescent depression.

\section{Key words}

Major depressive disorder • Pupil response - Central autonomic regulation/dysregulation $\bullet$ Emotional processing $\bullet$ Adolescent age

\section{Corresponding author}

N. Sekaninova, Department of Physiology and Biomedical Center Martin, Jessenius Faculty of Medicine in Martin, Comenius University in Bratislava, Mala Hora 4C, 03601 Martin, Slovak Republic. E-mail: nikola.sekaninova@gmail.com

\section{Introduction}

Interindividual differences in the responsivity of the stress response system to long-term stress exposition associated with altered sympathetic regulation may contribute to the risk for mental disorders (Mestanik et al. 2015, Tonhajzerova and Mestanik 2017). Major depressive disorder (MDD) as a negative affective state defined by emotion dysregulation with sustained negative affect and a persistent reduction in positive affect is associated with autonomic imbalance (Joormann and Gotlib 2010). In this context, pupil diameter is regulated by autonomic nervous system under central control mechanisms. Specifically, changes of the pupil size are determined by the tone of two opposing muscles, dilatator and constrictor. Pupillary constriction is mediated by constrictor under parasympathetic control regulated from Edinger-Westphal complex whereas 
pupillary dilation mediated by dilatator is regulated by sympathetic activity under posterior hypothalamic nuclei control (Steinhauer and Hakerem 1992), therefore, pupil dilation as an indicator of oculometric behaviour (Proudfoot et al. 2016) could be determined by increased sympathetic activity and by inhibition of parasympathetic activity.

Pupillary light reflex (PLR) - pupil constriction in response to illumination is mediated mainly by parasympathetic nervous system predominantly regulated at the subcortical level. Specifically, light falling on the retina activates the pretectal olivary nuclei with consequent activation of the Edinger-Westphal region resulting in generation of action potentials through the oculomotor nerves innervating constrictor muscle resulting in pupil constriction (McDougal and Gamlin 2015). Thus, corresponding constriction parameters are considered to be the most robust for detecting parasympathetic dysfunction (Hall and Chilcott 2018). In this aspect, our previous study pointed to altered PLR in the manner of resting parasympathetic hypoactivity in adolescent depression (Mestanikova et al. 2017).

Pupillary response is also included in emotional processing (Stone et al. 2016). Pupil dilation which begins shortly after picture onset and continues during whole affective picture viewing is mediated by increased sympathetic activity and is determined by emotional arousal (Bradley et al. 2017). Therefore, modulation of the pupil diameter during emotional picture viewing can provide an investigatory paradigm for studying emotional processing, which abnormalities are associated with depression (Burkhouse et al. 2017). Specifically, depressive patients tend to experience increased negative affect, reduced positive affect and attentional bias toward depression-relevant stimuli (Hankin et al. 2010). Thus, there is an assumption that biased processing of emotional stimuli may serve as a key of feature in the development and maintenance of depressive disorder.

From neurophysiological perspective, regarding the neural correlates of emotional processing, MDD is associated with increased activity within ventromedial prefrontal cortex/ventral anterior cingulate cortex and reduced activity regarding dorsomedial prefrontal cortex/dorsal anterior cingulate cortex resulting in dysregulation of limbic areas, particularly amygdala. Thus, increased reactivity of amygdala is not effectively downregulated by prefrontal cortex-mediated inhibition (Disner et al. 2011). Moreover, the anterior cingulate cortex (ACC) and amygdala are bi-directionally interconnected with locus coeruleus, a subcortical structure involved in physiological arousal, therefore, the activity of ACC as a part of a network of autonomic arousal control can be reflected in pupil diameter (Ebitz and Platt 2015, Costa and Rudebeck 2016).

Depressed adults exhibit greater pupil dilation to negative emotional stimuli compared to never depressed adults (Siegle et al. 2003), however, less is known in depressed youth. Silk et al. (2007) found that depressive adolescents exhibited less pupil dilation in response to negative words compared to never depressed youth. Contrary, Burkhouse et al. (2014) revealed that children of depressed mothers exhibited increased pupil dilation to sad faces compared to children of non-depressed mothers. Thus, the effect of emotionally valenced stimuli on pupil response in children and adolescents is still unclear. In this regard, eye-tracking and pupillometry represent novel non-invasive methods suitable for quantifying of central autonomic regulation/dysregulation influenced by emotional processing associated with MDD.

We hypothesized that MDD at adolescent age could be associated with dysfunctions in central autonomic regulation and emotional processing resulting in abnormal pupillary response. Therefore, the aim was to study changes in central autonomic regulation indexed by oculometric behaviour based on evaluation of pupil response to emotional stimuli in adolescents suffering from major depression.

\section{Methods}

\section{Ethics statement}

The study was approved by the Ethics Committee of Jessenius Faculty of Medicine in Martin, Comenius University in Bratislava in accordance with the Declaration of Helsinki (2000). All probands and their parents were thoroughly instructed about the study protocol and they gave written informed consent before participation in the study.

\section{Participants}

We have examined 20 adolescent patients suffering from major depressive disorder (MDD) (15 females, average age: $15.4 \pm 1.2$ years) and 20 gender and age matched healthy subjects (control group). The depressive patients were recruited from the inpatients admitted to Psychiatric Clinic of Jessenius Faculty of Medicine and University Hospital in Martin and they were examined during the first days of hospitalization 
prior to pharmacotherapy. The diagnosis of MDD, single episode without psychotic symptoms was classified by clinical investigation based on diagnostic interview by a child/adolescent psychiatrist according to Diagnostic and Statistical Manual of Mental Disorders, DSM-5 (American Psychiatric Association 2013). The subjects included in control group have never suffered from any mental disorder. Exclusion criteria for both MDD and control group were: underweight, overweight/obesity, history of neurological, cardiovascular, endocrinological, respiratory, metabolic or infectious diseases, smoking and alcohol or psychoactive drugs abuse. All participants had normal or corrected-to-normal vision. In addition, all probands were right-handed.

\section{Study protocol}

All participants were examined in psychophysiological laboratory (quiet darkened room) with minimization of stimuli under standard conditions (same light intensity, temperature: $22-23{ }^{\circ} \mathrm{C}$ ) in the morning after a normal breakfast. Subjects were instructed to sit comfortably in a special armchair after attaching and calibration of the recording device. After $15 \mathrm{~min}$ required for the adaptation to the examination room conditions, the participants remained in the sitting position (baseline phase). The continuous recording of the pupil diameter was performed in the following order during 5 phases: baseline, free-view task (3 blocks consisted of positive, neutral and negative pictures) and recovery phase (Fig. 1). Each phase lasted 5 min. After each phase the parameters of pupillary light reflex separately for both eyes were recorded using a handheld infrared optical scanner Pupillometer PLR-2000 (NeurOptics, USA).

\section{Experiment free-view task}

Participants were instructed that blocks of pictures would be displayed and that each picture should be scanned freely during the whole time it was on the screen. Emotional visual stimuli were selected from Nencki Affective Picture System (NAPS, Marchewka et al. 2014) consisting of 50 pleasant (mean pleasure/arousal=7.40/5.7), 50 neutral (mean pleasure/arousal $=5.2 / 3.3$ ) and 50 unpleasant (mean pleasure/arousal $=2.57 / 6.7$ ) pictures. All pictures have been standardized on the basis of following affective dimensions: valence (positive/negative), arousal (relaxed/excited) and approach-avoidance (tendency to approach/avoid a stimulus). Stimuli were displayed in a full-screen view for $6 \mathrm{~s}$ on a 17 inch screen placed at a distance of approximately $60 \mathrm{~cm}$ from the participant, were horizontal in orientation and portrayed people, faces, animals, objects and landscapes. The selected pictures were adjusted for luminance to control trivial luminance-related effects on pupil diameter responses. Pictures were presented to the participants in the same order: pleasant, neutral and unpleasant.

\section{Apparatus}

Pupillometric data were continuously recorded using a Mobile Eye-tracking Headset (Pupil labs, Germany) which consists of a video camera and an infrared light source that point to the participants' right eye. The recording is triggered after automatic 5-point calibration. Pupil diameter was sampled at $60 \mathrm{~Hz}$ (each $16.7 \mathrm{~ms})$. PLR parameters were tracked using a Pupillometer PLR-2000 (NeurOptics, USA) with a sampling frequency $32 \mathrm{~Hz}$ and accuracy $0.01 \mathrm{~mm}$. During PLR measurement probands were instructed to

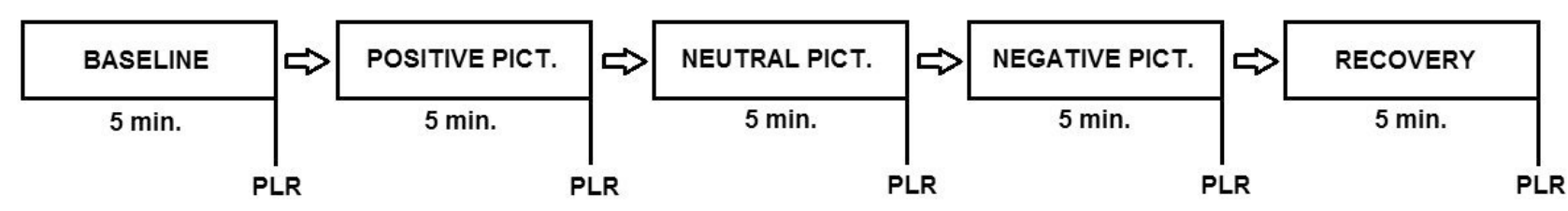

PLR measurement

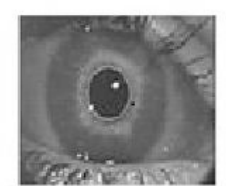

initial pupil size
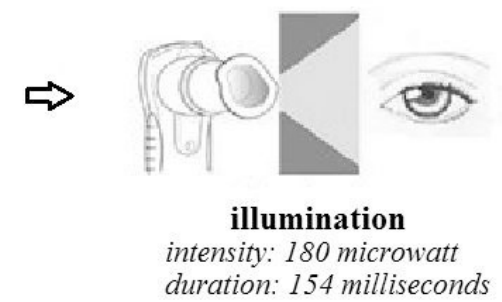

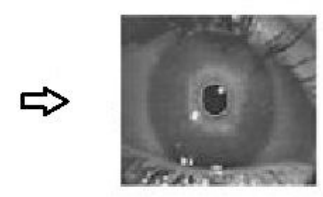

pupil size at the peak of constriction

Fig. 1. The scheme of study protocol. PLR - pupillary light reflex. 
keep their head straight, keep eyes opened without blinking and fix their gaze on a spot $3 \mathrm{~m}$ from a participant. Subsequently, the pupil margins were electronically detected by infrared illumination and then the pupil changes were continuously recorded during $5 \mathrm{~s}$ after light stimulus application. Pupillometry should not be measured when contact eye lenses are wearing or in the case of light-flushing, thus, all participants were asked not to wear contact eye lenses during participation on the study.

\section{Eye-tracking}

The pupil response according to each phase of study protocol was calculated as the mean change of pupil diameter after each phase onset using a Pupil Player Software (Pupil labs, Germany). Periods of data due to blink-related eye artefacts or missing data were automatically excluded and subsequently manually checked and, if necessary, corrected.

\section{Pupillary light reflex}

Evaluated parameters were: the initial value of pupil diameter in millimeters before light stimulus application (INIT), after illumination at the peak of constriction (END) and percentual change of the pupil diameter during constriction $($ DELTA $=($ INIT - END) / INIT $x \quad 100 \%$ ). Moreover, we assessed additional parameters of PLR dynamics: latency (LAT) - the onset time of the constriction after illumination, average constriction velocity (AVC, mm/s), maximum constriction velocity $(\mathrm{MCV}, \mathrm{mm} / \mathrm{s})$ and average dilation velocity $(\mathrm{ADV}, \mathrm{mm} / \mathrm{s})$. The parasympathetic-mediated parameters are LAT, DELTA, ACV and MCV, whereas INIT, END and ADV are mediated by both, sympathetic and parasympathetic, branches of autonomic nervous system (Hall and Chilcott 2018).

\section{Statistical analysis}

The data were analysed using statistical software package SYSTAT 10 (Cranes Software International Ltd, USA). The non-Gaussian/Gaussian distribution was ascertained by Shapiro-Wilk normality test. The KruskalWallis test was performed for data with non-parametric distribution and Student's unpaired $t$-test was used for data with parametric distribution for comparison between groups and eyes differences. Subsequently, Wilcoxon test for data with non-parametric distribution and Student's paired $t$-test for data with parametric distribution were used for comparison between emotional phases in each group. Data are expressed as mean \pm SEM. The probabilities $\mathrm{p}<0.05$ were considered as significant.

\section{Results}

\section{Comparison between depressive and control groups Right eye}

According to average pupil diameter, there was significantly greater pupil dilation during positive pictures free-view task in the depressive group compared to controls $(p=0.013)$, in accordance with significant greater pupil dilation indexed by initial pupil diameter before illumination (INIT) in the depressive group compared to the control group $(\mathrm{p}=0.031)$. No significant differences were found in average pupil diameter during remaining phases of the study protocol.

Regarding other PLR parameters, average and maximum constriction velocity (ACV, MCV, respectively) were significantly slower in baseline and during positive pictures free-view task in the depressive group compared to control group ( $\mathrm{p}=0.036, \mathrm{p}=0.050$; $\mathrm{p}=0.021, \mathrm{p}=0.015$, respectively). In addition, MCV was significantly slower in recovery phase in depressive patients compared to controls $(\mathrm{p}=0.045)$. Moreover, average dilation velocity (ADV) was significantly faster in negative pictures free-view task in the depressive group compared to controls $(p=0.042)$. No significant differences were found in the remaining parameters - the initial pupil diameter before light application (INIT), and after light illumination at the peak of the constriction (END), and constriction percentual change (DELTA).

\section{Left eye}

PLR parameter - constriction percentual change (DELTA) was significantly lower in the depressive group compared to the control group $(p=0.044)$. No significant between-group differences were found in the remaining parameters in assembling phases. All results are summarized in Tables 1 and 2.

\section{Comparison between left and right eyes}

The statistical analysis revealed no significant differences in the evaluated parameters between the left and right eye in alone depressive or control group.

Comparison between emotional visual stimuli free viewing phases (positive vs. neutral, positive vs. negative, neutral vs. negative pictures)

In the depressive group, the PLR parameters baseline pupil diameter (INIT) and pupil diameter at the peak of constriction (END) - were significantly greater to 
Table 1. Pupillary light reflex parameters.

\begin{tabular}{|c|c|c|c|c|}
\hline & \multicolumn{2}{|c|}{ Right eye } & \multicolumn{2}{|c|}{ Left eye } \\
\hline & Controls $(n=20)$ & $\operatorname{MDD}(n=20)$ & Controls $(n=20)$ & $\operatorname{MDD}(n=20)$ \\
\hline \multicolumn{5}{|l|}{ PLR baseline } \\
\hline $\operatorname{INIT}(\mathrm{mm})$ & $4.21 \pm 0.11$ & $4.54 \pm 0.15$ & $4.40 \pm 0.12$ & $4.61 \pm 0.18$ \\
\hline$E N D(\mathrm{~mm})$ & $3.05 \pm 0.09$ & $3.24 \pm 0.11$ & $3.23 \pm 0.10$ & $3.30 \pm 0.11$ \\
\hline DELTA (\%) & $-28.10 \pm 1.14$ & $-28.60 \pm 0.92$ & $-26.85 \pm 0.90$ & $-28.10 \pm 1.31$ \\
\hline $\operatorname{LAT}(s)$ & $0.22 \pm 0.01$ & $0.23 \pm 0.01$ & $0.23 \pm 0.00$ & $0.24 \pm 0.00$ \\
\hline$A C V(\mathrm{~mm} / \mathrm{s})$ & $-3.34 \pm 0.11$ & $-3.69 \pm 0.15^{*}$ & $-3.48 \pm 0.11$ & $-3.60 \pm 0.20$ \\
\hline$M C V(\mathrm{~mm} / \mathrm{s})$ & $-4.26 \pm 0.17$ & $-4.72 \pm 0.19 *$ & $-4.38 \pm 0.14$ & $-4.67 \pm 0.26$ \\
\hline$A D V(\mathrm{~mm} / \mathrm{s})$ & $1.45 \pm 0.08$ & $1.55 \pm 0.09$ & $1.44 \pm 0.08$ & $1.45 \pm 0.04$ \\
\hline \multicolumn{5}{|c|}{ PLR positive picture } \\
\hline $\operatorname{INIT}(\mathrm{mm})$ & $4.23 \pm 0.11$ & $4.61 \pm 0.16 *$ & $4.45 \pm 0.17$ & $4.73 \pm 0.16$ \\
\hline$E N D(m m)$ & $3.06 \pm 0.09$ & $3.25 \pm 0.10$ & $3.25 \pm 0.13$ & $3.33 \pm 0.11$ \\
\hline DELTA (\%) & $-27.45 \pm 0.88$ & $-29.20 \pm 1.09$ & $-26.85 \pm 0.90$ & $-28.90 \pm 1.03$ \\
\hline $\operatorname{LAT}(s)$ & $0.22 \pm 0.01$ & $0.23 \pm 0.01$ & $0.22 \pm 0.00$ & $0.23 \pm 0.01$ \\
\hline$A C V(\mathrm{~mm} / \mathrm{s})$ & $-3.38 \pm 0.13$ & $-3.83 \pm 0.18 *$ & $-3.55 \pm 0.14$ & $-3.79 \pm 0.15$ \\
\hline$M C V(\mathrm{~mm} / \mathrm{s})$ & $-4.29 \pm 0.15$ & $-4.88 \pm 0.23 *$ & $-4.45 \pm 0.17$ & $-4.79 \pm 0.21$ \\
\hline$A D V(\mathrm{~mm} / \mathrm{s})$ & $1.50 \pm 0.07$ & $1.59 \pm 0.08$ & $1.40 \pm 0.08$ & $1.37 \pm 0.06$ \\
\hline \multicolumn{5}{|c|}{ PLR neutral picture } \\
\hline $\operatorname{INIT}(\mathrm{mm})$ & $4.24 \pm 0.11$ & $4.53 \pm 0.17$ & $4.40 \pm 0.15$ & $4.61 \pm 0.19$ \\
\hline$E N D(\mathrm{~mm})$ & $3.04 \pm 0.09$ & $3.20 \pm 0.11$ & $3.23 \pm 0.12$ & $3.27 \pm 0.12$ \\
\hline$D E L T A(\%)$ & $-28.00 \pm 0.69$ & $-28.35 \pm 1.22$ & $-26.65 \pm 0.99$ & $-28.50 \pm 1.33$ \\
\hline $\operatorname{LAT}(s)$ & $0.22 \pm 0.00$ & $0.23 \pm 0.01$ & $0.22 \pm 0.01$ & $0.23 \pm 0.00$ \\
\hline$A C V(\mathrm{~mm} / \mathrm{s})$ & $-3.52 \pm 0.10$ & $-3.69 \pm 0.21$ & $-3.41 \pm 0.13$ & $-3.68 \pm 0.21$ \\
\hline$M C V(\mathrm{~mm} / \mathrm{s})$ & $-4.44 \pm 0.13$ & $-4.69 \pm 0.26$ & $-4.30 \pm 0.16$ & $-4.66 \pm 0.26$ \\
\hline$A D V(\mathrm{~mm} / \mathrm{s})$ & $1.44 \pm 0.07$ & $1.46 \pm 0.08$ & $1.36 \pm 0.08$ & $1.38 \pm 0.05$ \\
\hline \multicolumn{5}{|c|}{ PLR negative picture } \\
\hline$I N I T(\mathrm{~mm})$ & $4.25 \pm 0.13$ & $4.59 \pm 0.16$ & $4.45 \pm 0.15$ & $4.50 \pm 0.17$ \\
\hline END $(\mathrm{mm})$ & $3.09 \pm 0.10$ & $3.23 \pm 0.10$ & $3.28 \pm 0.12$ & $3.18 \pm 0.10$ \\
\hline DELTA $(\%)$ & $-27.45 \pm 0.84$ & $-29.35 \pm 1.16$ & $-26.00 \pm 0.94$ & $-28.80 \pm 1.32 \%$ \\
\hline $\operatorname{LAT}(s)$ & $0.22 \pm 0.00$ & $0.22 \pm 0.01$ & $0.23 \pm 0.01$ & $0.23 \pm 0.01$ \\
\hline$A C V(\mathrm{~mm} / \mathrm{s})$ & $-3.51 \pm 0.14$ & $-3.77 \pm 0.17$ & $-3.41 \pm 0.14$ & $-3.70 \pm 0.21$ \\
\hline$M C V(\mathrm{~mm} / \mathrm{s})$ & $-4.46 \pm 0.18$ & $-4.72 \pm 0.23$ & $-4.32 \pm 0.18$ & $-4.67 \pm 0.27$ \\
\hline$A D V(\mathrm{~mm} / \mathrm{s})$ & $1.37 \pm 0.07$ & $1.58 \pm 0.07 *$ & $1.29 \pm 0.07$ & $1.38 \pm 0.08$ \\
\hline \multicolumn{5}{|c|}{ PLR recovery phase } \\
\hline $\operatorname{INIT}(\mathrm{mm})$ & $4.10 \pm 0.14$ & $4.39 \pm 0.14$ & $4.20 \pm 0.16$ & $4.37 \pm 0.16$ \\
\hline END (mm) & $2.97 \pm 0.09$ & $3.13 \pm 0.09$ & $3.10 \pm 0.11$ & $3.09 \pm 0.10$ \\
\hline DELTA (\%) & $-27.60 \pm 0.84$ & $-28.40 \pm 1.42$ & $-26.10 \pm 1.15$ & $-28.85 \pm 1.24$ \\
\hline$L A T(s)$ & $0.21 \pm 0.01$ & $0.23 \pm 0.01$ & $0.22 \pm 0.00$ & $0.23 \pm 0.01$ \\
\hline$A C V(\mathrm{~mm} / \mathrm{s})$ & $-3.35 \pm 0.15$ & $-3.65 \pm 0.19$ & $-3.36 \pm 0.16$ & $-3.64 \pm 0.20$ \\
\hline$M C V(\mathrm{~mm} / \mathrm{s})$ & $-4.16 \pm 0.17$ & $-4.66 \pm 0.24 *$ & $-4.19 \pm 0.21$ & $-4.64 \pm 0.25$ \\
\hline$A D V(\mathrm{~mm} / \mathrm{s})$ & $1.34 \pm 0.10$ & $1.43 \pm 0.07$ & $1.40 \pm 0.06$ & $1.44 \pm 0.06$ \\
\hline
\end{tabular}

MDD - major depressive disorder, INIT - pupil diameter before illumination, END - pupil diameter after illumination at the peak of constriction, DELTA - percentual change of the pupil diameter, LAT - time of the constriction onset after illumination, ACV - average constriction velocity, MCV - maximum constriction velocity, ADV - average dilation velocity. Values are expressed as mean \pm SEM. Probabilities $p<0.05$ were considered as significant. ${ }^{*} p<0.05$. 
Table 2. Average pupil diameter assessed by eye-tracking.

\begin{tabular}{lcc}
\hline & & Right eye \\
& Controls $(\mathrm{n}=20)$ & MDD $(\mathrm{n}=20)$ \\
\hline Average pupil diameter $(\mathbf{m})$ & & \\
Baseline & $3.80 \pm 0.15$ & $3.91 \pm 0.14$ \\
Positive pictures & $3.84 \pm 0.09$ & $\mathbf{4 . 5 2} \pm \mathbf{0 . 2 8 *}$ \\
Neutral pictures & $3.83 \pm 0.10$ & $4.49 \pm 0.27$ \\
Negative pictures & $3.99 \pm 0.10$ & $4.38 \pm 0.22$ \\
Recovery phase & $3.68 \pm 0.09$ & $4.13 \pm 0.19$ \\
\hline
\end{tabular}

MDD - major depressive disorder. Values are expressed as mean \pm SEM. Probabilities $p<0.05$ were considered as significant. ${ }^{*} p<0.05$.

positive pictures free-viewing compared to negative ones on the left eye $(p=0.002, p=0.015$, respectively). No significant differences were found in the remaining parameters. All results are summarized in Tables 3 and 4.

\section{Discussion}

The major findings of this study regarding the central autonomic activity and emotional processing assessed by spontaneous changes of pupil diameter and its response to light - pupillary light reflex (PLR) are following: 1) altered PLR in adolescent depressive patients compared to controls indicating parasympathetic hypoactivity associated with tendency to increased sympathetic activity; 2) discrepancies in pupil response to illumination between left and right eye during different phases of the study protocol (namely baseline, positive and negative pictures free-viewing and recovery) in adolescents suffering from major depressive disorder; 3) tendency to pronounced pupil dilation in the depressive group compared to controls, however, only average pupil diameter during positive pictures freeviewing reached significance; 4) significantly greater pupil diameter indexed by PLR parameters (INIT, END) to positive pictures free-viewing compared to negative ones on the left eye only in the depressive group. In this context, we supposed that our findings of discrete differences in pupil response in adolescent depressive patients could indicate subtle disturbance in central autonomic integrity influenced by emotional reactivity.

PLR, i.e. constriction and subsequent dilation of the pupil to illumination is mediated by parasympathetic and sympathetic nervous system. Thus, different PLR parameters can be used as potential biomarkers for central complex sympathovagal regulation. Specifically, PLR constriction parameters robustly detecting parasympathetic activity such as maximum constriction velocity and maximum constriction amplitude reflecting response latency and maximum pupil constriction reflect the action of constrictor muscle, function of retinal photoreceptors and time consumed in afferent and efferent pathway. In contrast, pupil dilation following the light stimulus mediated by sympathetic nervous system corresponds to the PLR recovery phase indexed by average dilation velocity (Hall and Chilcott 2018). In this context, our main findings of right eye-linked slower average (ACV) and maximum constriction velocity (MCV) in the baseline, and positive pictures free-view task, and slower MCV alone in the recovery phase could point to deficient central parasympathetic activity in adolescent major depressive disorder. These findings are in accordance with our previous study revealing resting central autonomic dysregulation assessed by PLR in adolescent depression (Mestanikova et al. 2017). Exactly, primary neural circuit implicated in PLR is regulated at the subcortical level with the crucial role of the parasympathetic-linked Edinger-Westphal nucleus that activation leads to pupil constriction via sphincter muscle (McDougal and Gamlin 2015). Importantly, this circuit is influenced by several inhibitory inputs. Specifically, the Edinger-Westphal nucleus activity is inhibited by the sympathetic-linked subcortical brain structure locus coeruleus. Thus, higher sympathetic activity leads to excited inhibitory mechanism on Edinger-Westphal nucleus resulting in pupil dilation (Wilhelm et al. 1999). In this aspect, the potential pathomechanism of abnormal PLR in the manner of slower ACV and MCV in adolescent depression could be characterized by increased central sympathetic activity associated with parasympathetic hypoactivity at the subcortical level. Furthermore, Edinger-Westphal nucleus is also influenced by inhibitory inputs from direct cortical and 
Table 3. Comparison of PLR parameters between emotional phases of the protocol.

\begin{tabular}{|c|c|c|c|c|}
\hline \multirow[b]{2}{*}{ PLR parameters } & \multicolumn{2}{|c|}{ Right eye } & \multicolumn{2}{|c|}{ Left eye } \\
\hline & Controls $(n=20)$ & MDD $(n=20)$ & Controls $(n=20)$ & MDD $(n=20)$ \\
\hline \multicolumn{5}{|c|}{ Positive vs. neutral picture } \\
\hline $\operatorname{INIT}(\mathrm{mm})$ & $\begin{array}{c}4.23 \pm 0.11 \mathrm{vs} . \\
4.24 \pm 0.11\end{array}$ & $\begin{array}{c}4.61 \pm 0.16 \mathrm{vs} . \\
4.53 \pm 0.17\end{array}$ & $\begin{array}{c}4.45 \pm 0.17 \text { vs. } \\
4.40 \pm 0.15\end{array}$ & $\begin{array}{c}4.73 \pm 0.16 \text { vs. } \\
4.61 \pm 0.19\end{array}$ \\
\hline$E N D(m m)$ & $\begin{array}{c}3.06 \pm 0.09 \text { vs. } \\
3.04 \pm 0.09\end{array}$ & $\begin{array}{c}3.25 \pm 0.10 \text { vs. } \\
3.20 \pm 0.11\end{array}$ & $\begin{array}{c}3.25 \pm 0.13 \text { vs. } \\
3.23 \pm 0.12\end{array}$ & $\begin{array}{c}3.33 \pm 0.11 \text { vs. } \\
3.27 \pm 0.12\end{array}$ \\
\hline DELTA $(\%)$ & $\begin{array}{c}-27.45 \pm 0.84 \mathrm{vs} \\
-28.00 \pm 0.69\end{array}$ & $\begin{array}{c}-29.20 \pm 1.09 \text { vs. } \\
-28.35 \pm 1.22\end{array}$ & $\begin{array}{c}-26.85 \pm 0.90 \text { vs. } \\
-26.65 \pm 0.99\end{array}$ & $\begin{array}{c}-28.90 \pm 1.03 \text { vs. } \\
-28.50 \pm 1.33\end{array}$ \\
\hline $\operatorname{LAT}(s)$ & $\begin{array}{c}0.22 \pm 0.00 \text { vs. } \\
0.22 \pm 0.00\end{array}$ & $\begin{array}{c}0.23 \pm 0.01 \mathrm{vs} . \\
0.23 \pm 0.01\end{array}$ & $\begin{array}{c}0.22 \pm 0.00 \text { vs. } \\
0.22 \pm 0.01\end{array}$ & $\begin{array}{c}0.23 \pm 0.01 \mathrm{vs} \\
0.23 \pm 0.00\end{array}$ \\
\hline$A C V(\mathrm{~mm} / \mathrm{s})$ & $\begin{array}{c}-3.38 \pm 0.13 \text { vs. } \\
-3.52 \pm 0.10\end{array}$ & $\begin{array}{c}-3.83 \pm 0.18 \text { vs. } \\
-3.69 \pm 0.21\end{array}$ & $\begin{array}{c}-3.55 \pm 0.14 \text { vs. } \\
-3.41 \pm 0.13\end{array}$ & $\begin{array}{c}-3.79 \pm 0.15 \mathrm{vs} \\
-3.68 \pm 0.21\end{array}$ \\
\hline$M C V(\mathrm{~mm} / \mathrm{s})$ & $\begin{array}{c}-4.29 \pm 0.15 \mathrm{vs} \\
-4.44 \pm 0.13\end{array}$ & $\begin{array}{c}-4.88 \pm 0.23 \text { vs. } \\
-4.69 \pm 0.26\end{array}$ & $\begin{array}{c}-4.45 \pm 0.17 \text { vs. } \\
-4.30 \pm 0.16\end{array}$ & $\begin{array}{c}-4.79 \pm 0.21 \text { vs. } \\
-4.66 \pm 0.26\end{array}$ \\
\hline$A D V(\mathrm{~mm} / \mathrm{s})$ & $\begin{array}{c}1.50 \pm 0.07 \mathrm{vs} . \\
1.44 \pm 0.07\end{array}$ & $\begin{array}{c}1.59 \pm 0.08 \mathrm{vs} . \\
1.46 \pm 0.08\end{array}$ & $\begin{array}{c}1.40 \pm 0.08 \mathrm{vs} \\
1.36 \pm 0.08\end{array}$ & $\begin{array}{c}1.37 \pm 0.06 \mathrm{vs} \\
1.38 \pm 0.05\end{array}$ \\
\hline \multicolumn{5}{|c|}{ Positive vs. negative picture } \\
\hline $\operatorname{INIT}(\mathrm{mm})$ & $\begin{array}{c}4.23 \pm 0.11 \mathrm{vs} . \\
4.25 \pm 0.13\end{array}$ & $\begin{array}{c}4.61 \pm 0.16 \mathrm{vs} . \\
4.59 \pm 0.16\end{array}$ & $\begin{array}{c}4.45 \pm 0.17 \text { vs. } \\
4.45 \pm 0.15\end{array}$ & $\begin{array}{c}4.73 \pm 0.16 \text { vs. } \\
4.50 \pm 0.17 *\end{array}$ \\
\hline$E N D(m m)$ & $\begin{array}{c}3.06 \pm 0.09 \text { vs. } \\
3.09 \pm 0.10\end{array}$ & $\begin{array}{c}3.25 \pm 0.10 \text { vs. } \\
3.23 \pm 0.10\end{array}$ & $\begin{array}{c}3.25 \pm 0.13 \text { vs. } \\
3.28 \pm 0.12\end{array}$ & $\begin{array}{c}3.33 \pm 0.11 \text { vs. } \\
3.18 \pm 0.10 *\end{array}$ \\
\hline DELTA $(\%)$ & $\begin{array}{c}-27.45 \pm 0.88 \text { vs. } \\
-27.45 \pm 0.84\end{array}$ & $\begin{array}{c}-29.20 \pm 1.01 \mathrm{vs} . \\
-29.35 \pm 1.16\end{array}$ & $\begin{array}{c}-26.85 \pm 0.90 \mathrm{vs} . \\
-26.00 \pm 0.94\end{array}$ & $\begin{array}{c}-28.90 \pm 1.03 \text { vs. } \\
-28.80 \pm 1.32\end{array}$ \\
\hline$L A T(s)$ & $\begin{array}{c}0.22 \pm 0.00 \text { vs. } \\
0.22 \pm 0.00\end{array}$ & $\begin{array}{c}0.23 \pm 0.01 \text { vs. } \\
0.22 \pm 0.01\end{array}$ & $\begin{array}{c}0.22 \pm 0.00 \text { vs. } \\
0.23 \pm 0.01\end{array}$ & $\begin{array}{c}0.23 \pm 0.01 \mathrm{vs} . \\
0.23 \pm 0.01\end{array}$ \\
\hline$A C V(\mathrm{~mm} / \mathrm{s})$ & $\begin{array}{c}-3.38 \pm 0.13 \text { vs. } \\
-3.51 \pm 0.14\end{array}$ & $\begin{array}{c}-3.83 \pm 0.18 \text { vs. } \\
-3.77 \pm 0.17\end{array}$ & $\begin{array}{c}-3.55 \pm 0.14 \text { vs. } \\
-3.41 \pm 0.14\end{array}$ & $\begin{array}{c}-3.79 \pm 0.15 \text { vs. } \\
-3.70 \pm 0.21\end{array}$ \\
\hline$M C V(\mathrm{~mm} / \mathrm{s})$ & $\begin{array}{c}-4.29 \pm 0.15 \mathrm{vs} \\
-4.46 \pm 0.18\end{array}$ & $\begin{array}{c}-4.88 \pm 0.23 \text { vs. } \\
-4.72 \pm 0.23\end{array}$ & $\begin{array}{c}-4.45 \pm 0.17 \text { vs. } \\
-4.32 \pm 0.18\end{array}$ & $\begin{array}{c}-4.79 \pm 0.21 \text { vs. } \\
-4.67 \pm 0.27\end{array}$ \\
\hline$A D V(\mathrm{~mm} / \mathrm{s})$ & $\begin{array}{c}1.50 \pm 0.07 \text { vs. } \\
1.37 \pm 0.07\end{array}$ & $\begin{array}{c}1.59 \pm 0.08 \text { vs. } \\
1.58 \pm 0.07\end{array}$ & $\begin{array}{c}1.40 \pm 0.08 \text { vs. } \\
1.29 \pm 0.07\end{array}$ & $\begin{array}{c}1.37 \pm 0.06 \text { vs. } \\
1.38 \pm 0.08\end{array}$ \\
\hline \multicolumn{5}{|c|}{ Neutral vs. negative picture } \\
\hline $\operatorname{INIT}(\mathrm{mm})$ & $\begin{array}{c}4.24 \pm 0.11 \mathrm{vs} . \\
4.25 \pm 0.13\end{array}$ & $\begin{array}{c}4.53 \pm 0.17 \mathrm{vs} . \\
4.59 \pm 0.16\end{array}$ & $\begin{array}{c}4.40 \pm 0.15 \text { vs. } \\
4.45 \pm 0.15\end{array}$ & $\begin{array}{c}4.61 \pm 0.19 \mathrm{vs} . \\
4.50 \pm 0.17\end{array}$ \\
\hline $\operatorname{END}(\mathrm{mm})$ & $\begin{array}{c}3.04 \pm 0.09 \text { vs. } \\
3.09 \pm 0.10\end{array}$ & $\begin{array}{c}3.20 \pm 0.11 \text { vs. } \\
3.23 \pm 0.10\end{array}$ & $\begin{array}{c}3.23 \pm 0.12 \text { vs. } \\
3.28 \pm 0.12\end{array}$ & $\begin{array}{c}3.27 \pm 0.12 \text { vs. } \\
3.18 \pm 0.10\end{array}$ \\
\hline DELTA $(\%)$ & $\begin{array}{c}-28.00 \pm 0.69 \text { vs. } \\
-27.45 \pm 0.84\end{array}$ & $\begin{array}{c}-28.35 \pm 1.22 \text { vs. } \\
-29.35 \pm 1.16\end{array}$ & $\begin{array}{c}-26.65 \pm 0.99 \text { vs. } \\
-26.00 \pm 0.94\end{array}$ & $\begin{array}{c}-28.50 \pm 1.33 \text { vs. } \\
-28.80 \pm 1.32\end{array}$ \\
\hline$L A T(s)$ & $\begin{array}{c}0.22 \pm 0.00 \mathrm{vs} \\
0.22 \pm 0.00\end{array}$ & $\begin{array}{c}0.23 \pm 0.01 \mathrm{vs} \\
0.22 \pm 0.01\end{array}$ & $\begin{array}{c}0.22 \pm 0.01 \text { vs. } \\
0.23 \pm 0.01\end{array}$ & $\begin{array}{c}0.23 \pm 0.00 \mathrm{vs} \\
0.23 \pm 0.01\end{array}$ \\
\hline$A C V(\mathrm{~mm} / \mathrm{s})$ & $\begin{array}{c}-3.52 \pm 0.10 \mathrm{vs} . \\
-3.51 \pm 0.14\end{array}$ & $\begin{array}{c}-3.69 \pm 0.21 \mathrm{vs} \\
-3.77 \pm 0.17\end{array}$ & $\begin{array}{c}-3.41 \pm 0.13 \text { vs. } \\
-3.41 \pm 0.14\end{array}$ & $\begin{array}{c}-3.68 \pm 0.21 \mathrm{vs} \\
-3.70 \pm 0.21\end{array}$ \\
\hline$M C V(\mathrm{~mm} / \mathrm{s})$ & $\begin{array}{c}-4.44 \pm 0.13 \text { vs. } \\
-4.46 \pm 0.18\end{array}$ & $\begin{array}{c}-4.69 \pm 0.26 \text { vs. } \\
-4.72 \pm 0.23\end{array}$ & $\begin{array}{c}-4.30 \pm 0.16 \text { vs. } \\
-4.32 \pm 0.18\end{array}$ & $\begin{array}{c}-4.66 \pm 0.26 \text { vs. } \\
-4.67 \pm 0.27\end{array}$ \\
\hline$A D V(\mathrm{~mm} / \mathrm{s})$ & $\begin{array}{c}1.44 \pm 0.07 \text { vs. } \\
1.37 \pm 0.07\end{array}$ & $\begin{array}{c}1.46 \pm 0.08 \mathrm{vs} . \\
1.58 \pm 0.07\end{array}$ & $\begin{array}{c}1.36 \pm 0.08 \mathrm{vs} \\
1.29 \pm 0.07\end{array}$ & $\begin{array}{c}1.38 \pm 0.05 \mathrm{vs} \\
1.38 \pm 0.08\end{array}$ \\
\hline
\end{tabular}

MDD - major depressive disorder, INIT - pupil diameter before illumination, END - pupil diameter after illumination at the peak of constriction, DELTA - percentual change of the pupil diameter, LAT - time of the constriction onset after illumination, ACV - average constriction velocity, MCV - maximum constriction velocity, ADV - average dilation velocity. Values are expressed as mean \pm SEM. Probabilities $\mathrm{p}<0.05$ were considered as significant. ${ }^{*} \mathrm{p}<0.05$. 
Table 4. Comparison of average pupil diameter between emotional phases of the protocol.

\begin{tabular}{llc}
\hline & Right eye & MDD (n=20) \\
\hline Average pupil diameter (mm) & & \\
Positive vs. neutral pictures & $3.84 \pm 0.09$ vs. $3.83 \pm 0.10$ & $4.52 \pm 0.28$ vs. $4.49 \pm 0.27$ \\
Positive vs. negative pictures & $3.84 \pm 0.09$ vs. $3.99 \pm 0.10$ & $4.52 \pm 0.28$ vs. $4.38 \pm 0.22$ \\
Neutral vs. negative pictures & $3.83 \pm 0.10$ vs. $3.99 \pm 0.10$ & $4.49 \pm 0.27$ vs. $4.38 \pm 0.22$ \\
\hline
\end{tabular}

MDD - major depressive disorder. Values are expressed as mean \pm SEM. Probabilities $p<0.05$ were considered as significant.

indirect cortico-thalamic-hypothalamic pathways (Steinhauer and Hakerem 1992). Recent studies suggest prefrontal cortex hypoactivity associated with frontal asymmetry in depressive patients (e.g. Grünewald et al. 2018), therefore, we suggest that altered PLR found in our adolescent depressive patients could be related to abnormal prefrontal cortex functioning associated with decreased inhibitory effect on parasympathetic oculomotor center.

From psychophysiological aspect, pupil response is modulated by emotionally evocative stimuli in the manner of pupil dilation in response to greater emotional intensity regardless of valence (Bradley et al. 2017) providing thus reliable index of locus coeruleus activity associated with emotional processes (Joshi et al. 2016). Moreover, locus coeruleus involved in physiological arousal is bi-directionally interconnected with amygdala as well as anterior cingulate cortex (ACC) - the key structures included in emotional processing. Additionally, ACC as a part of a network of autonomic arousal control can be reflected in pupil diameter (Ebitz and Platt 2015, Costa and Rudebeck 2016). Further, emotional processing is related to top-down cortical prefrontal control on the subcortical amygdala and amygdalaprefrontal cortex functional connectivity (Beauchaine 2015). Notably, the Edinger-Westphal nucleus activity is also modulated from cortical (prefrontal cortex) as well as subcortical (amygdala) brain regions interconnections (Dos Santos et al. 2015, Li et al. 2018). Taken together, emotion-linked modulation of pupil response results from complex cortical and subcortical regulatory inputs mediated by sympathetic and parasympathetic pathways.

However, the pupil response to emotional stimuli in children and adolescents suffering from major depression is still under extensive debate. For example, Silk et al. (2007) showed diminished pupil dilation to negative words in depressive children, whereas Burkhouse et al. (2014) revealed increased pupil dilation to sad faces in children of depressive mothers compared to children of non-depressed mothers. Our findings revealed increased baseline pupil diameter (INIT) and pupil diameter at the peak of constriction (END) in response to positive emotional visual stimuli and diminished late pupil response to negative emotional visual stimuli in the depressive group compared to the control group, and increased INIT and END regarding positive vs. negative emotional visual stimuli comparison only in the depressive group, indicating thus abnormal cognitive-affective regulatory processing of emotions in depressive adolescents.

Several explanations are assumed. Firstly, we suppose that sympathetic overexcitation indexed by greater INIT and END indices in response to positive pictures free-viewing may be explained as a consequence of the subjective perception of the positive pictures as more irritating stimulus leading to "internal stress reaction" associated with sympathetic activation in adolescent depression. In other words, negative emotional pictures appear to be more synchronous with altered subjective emotional feelings evoked by depressive disorder at adolescent age. Additionally, decreased cognitive-affective processing linked to depression may reflect emotional avoidance, affective blunting or overregulating as maladaptive regulatory strategies of negative emotions which are typical for depressive disorder (Silk et al. 2003). From neurophysiological aspect, depression-linked blunted amygdala reactivity could represent a potential pathomechanism leading to different pupil response to positive vs. negative emotional stimuli in adolescent depression (Thomas et al. 2001). Further, the disruption of inhibitory function of prefrontal and anterior cingulate cortices in emotion processing could be another important pathomechanism of depression-linked altered pupil response to emotional stress (Silk et al. 2007).

In addition, mid-to-late puberty adolescents 
more disengage their attention from the given task and could be also more bored or might demonstrate improved skills in refocusing attention away from emotional stimuli (Silk et al. 2009). It can explain our results of pronounced pupil response to positive stimuli compared to negative stimuli following positive ones in the free-view task. Another possible explanation of less pupil dilation in response to negative pictures viewing suggests that highly salient emotional information in depressionvulnerable individuals may overload their ability to process it (Graur and Siegle 2013).

Finally, major depressive disorder is associated with more sustained pupil response to emotional stimuli. In this context, sustained processing of briefly presented emotional stimuli could be associated with the tendency to rumination, i.e. maladaptive emotion regulation strategy, that may occur minutes, hours or even days after the stimulus arises (Siegle et al. 2003). Moreover, some studies indicate that depressive rumination is associated with parasympathetic withdrawal (Ottaviani et al. 2009). Therefore, our finding of altered PLR in recovery phase following negative pictures viewing task in adolescent MDD patients could be related to predisposition for depressive rumination as an emotional regulation strategy in processing negative material associated with parasympathetic underactivity.

It seems that our findings of altered PLR to emotional visual stimuli (positive, negative) could indicate depression-linked disturbance of brain regions important for emotional processing. These areas overlap with crucial structures involved in central autonomic network, as an important integral system of complex central autonomic regulation influencing peripheral effectors' responses (Benarroch 1993). From this perspective, our findings of altered parasympatheticlinked PLR parameters are in accordance with our previous studies pointing to diminished cardiac vagal control in adolescent depression (Tonhajzerova et al. 2010, Tonhajzerova et al. 2012, Mestanikova et al. 2019).

It is important to note possible relation of between-eyes differences to hemispheric lateralization of valence processing as well as autonomic control. The most recent hypothesis regarding the lateralization of emotions is the approach - withdrawal hypothesis stating that the left prefrontal cortex is associated with intention, planning and regulation that are important for approach behaviors, whereas the right prefrontal cortex is linked to behavioural inhibition important for withdrawal (Pereira and Khan 2017). This hypothesis overlaps with the valence hypothesis reporting that regions of the left hemisphere are specialized for the processing of positive stimuli while processing of negative stimuli involves the right hemisphere (Davidson and Tomarken 1989). According to these theories, depression could be associated with a stronger relationship to the frontal right hemisphere. Moreover, the right-hemisphere hyperactivity in depression may be due to dysfunction in the inhibitory system (Pereira and Khan 2017) and reduced left frontal activity in major depressive disorder may be associated with decreased response of striatum to positive stimuli (Bruder et al. 2017). However, brain lateralization of emotions may be more complex and region-specific than it is proposed.

Regarding the cortical lateralization of autonomic control, there is an evidence for right cerebral hemisphere dominance in sympathetic pupillary control, whereas parasympathetic activity seems to be under left hemisphere influence. Interestingly, pupillary response might be also influenced by extensive bilateral inputs from cortical as well as subcortical structures (Burtis et al. 2014). In this aspect, eye dominance associated with hand preference (Bourassa et al. 1996) shows functional laterality due to the dominance of one hemisphere (Gündoğan et al. 2009). Specifically, the right eye dominance is associated with functional brain laterality regarding the left hemisphere dominance (Gündoğan et al. 2009). With respect to pupillary response evaluated by PLR parameters there are significant differences between the left and right eye suggesting lateralization of cortical centers regulating autonomic nervous system (ANS) activity (Bär et al. 2005). Thus, asymmetrical activation of right and left hemispheres differentially modifies the ANS reactivity. Specifically, each side of retina primarily projects to the ipsilateral hemisphere's geniculocalcarine system, however, there is also evidence of the entire retina and the retinocollicular pathway projections to the contralateral superior colliculus (Perry and Cowey 1984). In addition, tectoreticular fibers from colliculus project to the mesencephalic reticular formation with more abundant ipsilateral fibers and each colliculus stimulation leads to ipsilateral hemispheric arousal response (Jefferson 1958, Truex and Carpenter 1964). Taken together, each superior colliculus seems to be able to activate the ipsilateral hemisphere via the tectoreticular system. In this context, monocular visual input into the brain may be able to asymmetrically activate the cerebral hemispheres.

Based on studies demonstrating right-left hemispheric asymmetries in autonomic control and 
potential effect of monocular viewing on hemispheric activation, it is assumed that occlusion of one eye (left) may reduce the activation of the right superior colliculus inducing relative inactivation of the right hemisphere preferentially mediating sympathetic activity. Thus, left eye occlusion may lead to relative pupillary constriction and vice versa, right eye occlusion with relative reduced activation of left hemisphere believed to be mediating parasympathetic activity may induce pupillary dilation (i.e. Hilz et al. 2001). However, it is still unclear whether the pupillary diameter change results from deactivation of the colliculus-hemisphere from contralateral eye patching or activation of the opposing hemisphere from viewing eye, or combination of both via interhemispheric inhibition through callosal communication. Thus, respiratory hippus variability (RHV) - respiratory frequency rhythmic oscillations of pupil diameter related to parasympathetic activity may provide a further insight into the relative independent contribution of parasympathetic activity to pupil size. In this aspect, detected pupillary right vs. left eye viewing asymmetries without simultaneous RHV alterations may suggest primarily sympathetically-linked pupillary changes. Contrary, RHV asymmetries reflecting pupillary constriction suggest parasympathetically-linked effect of monocular viewing (Burtis et al. 2014). It seems that the question whether the right eye dominance associated with functional laterality regarding left hemisphere dominance may influence the pupillary response to the light remains still discussed. Further research based on simultaneous continual recording of pupil changes (resting as well as in response to light), RHV in correlation with other noninvasive methods (e.g. HRV) is needed to elucidate the relations between eye-dominance and PLR.

Additionally, gender differences in hemispheric organization could play an important role. Heller (1993) suggested that the tendency to activate the left as opposed to right hemisphere among females may be associated with a neuropsychological vulnerability toward depression. Moreover, a meta-analysis based on neuroimaging studies indicated that the majority of gender differences in brain activation to emotional stimuli favoring women were found for negative emotions (Stevens and Hamann 2012). Therefore, it is unclear if our findings of different pupil responses in adolescent major depression from the left and right eye to emotional stimuli could be predominantly related to lateralization of cortical centers regulating autonomic activity and valence processing, as well. Future research based on concurrent pupillary and functional imaging studies in proportional female/male representation could help to resolve this question.

Importantly, PLR developmental changes as a noninvasive ocular predictor for central ANS integrity should be also taken into consideration. Recent study regarding age-period from 1 to 18 years revealed that both maximum and minimum pupil sizes were shown to increase until 11 years of age with subsequent plateau. Moreover, sympathetic PLR parameters such as dilation velocity showed a slight increase over the pediatric age range (from 1 to 18 years), whereas parasympatheticallymediated maximum constriction velocity decreased (Brown et al. 2015). In this aspect, these changes can reflect not only changes in eye size, but also developmental changes in PLR reflecting autonomic regulatory mechanisms. Specifically, PLR is well-known neurological process based on brisk and transient constriction of the pupil to fast increases in the light flux regulated by two cephalic ANS sympathetic and parasympathetic divisions. Thus, sympathetic activity may act through an increased central sympathetic inhibition of the Edinger-Westphal nucleus when light stimulus is received by the system. In this way, parameters reflecting parasympathetic activity (mainly MCV, maximum and relative constriction amplitude MCA, RCA) can be indirectly modulated by inhibition of sympathetic activity (Lobato-Rincón et al. 2014). Moreover, it is assumed that age-linked changes in pupilloconstrictor Edinger-Westphal nucleus are adjusted by an inhibitory projection from a number of cortical areas (Lobato-Rincón et al. 2014, Hall and Chilcott 2018). The inhibitory function crucial for organism functioning depends on age (Porges 2009, Porges and Furman 2011), therefore, we assume that maturation of the complex autonomic integrity could significantly influence pupillary response to light.

In contrast, Daluwatte et al. (2013) did not reveal age effect on PLR in healthy 8-15-year-old children, but age-linked dependence was significant on other effector - cardiac autonomic regulation indexed by heart rate variability (HRV). Similarly, our previous studies revealed developmental changes in cardiovascular autonomic reflex control in healthy adolescents from 15 to 19 years (Tonhajzerova et al. 1999, Tonhjazerova et al. 2002). Thus, PLR and HRV may provide complementary assessment of different aspects of the overall autonomic regulation from developmental aspect (Daluwatte et al. 2013). 


\section{Limitations}

Limitation of our study is a relatively small sample size, therefore, future studies including larger sample size with respect to gender are needed to validate our findings. Another limitation is that spontaneous changes of pupil diameter were recorded only from right eye, thus, it is not possible to assess potential differences in average pupil diameter between the left and right eye. Further, pupillometry provides information about central autonomic regulation, whereas information about peripheral effectors' regulation is limited. In this context, peripheral effectors' responses should be evaluated by other noninvasive methods, e.g. heart rate or blood pressure variability, mediated by both branches of ANS.

\section{Conclusions}

Our study revealed altered right-eye-linked pupillary light reflex to emotional visual stimuli indicating potential central autonomic dysregulation in the manner of parasympathetic hypoactivity associated with relative sympathetic dominance influenced by impairments in emotional processing already in adolescent major depressive disorder. In addition, between-eyes differences may be related to hemispheric lateralization of autonomic activity as well as valence processing in adolescent depression.

\section{Conflict of Interest}

There is no conflict of interest.

\section{Acknowledgements}

This work was supported by VEGA 1/0044/18, the project „Biomedical Center Martin“ ITMS code: 26220220187, co-financed from EU sources and the project „European Center of Excellence for Perinatological Research“ No. 26220120036.

\section{References}

AMERICAN PSYCHIATRIC ASSOCIATION: Depressive disorders. In: Diagnostic and Statistical Manual of Mental Disorders DSM-5. Fifth Edition. American Psychiatric Association, Arlington, 2013, pp 155-188.

BÄR KJ, BOETTGER MK, TILL S, DOLICEK J, SAUER H: Lateralization of pupillary light reflex parameters. Clin Neurophysiol 116: 790-798, 2005.

BEAUCHAINE TP: Future directions in emotion dysregulation and youth psychopathology. $J$ Clin Child Adolesc Psychol 44: 875-896, 2015.

BENARROCH EE: The central autonomic network: functional organization, dysfunction, and perspective. Mayo Clin Proc 68: 988-1001, 1993.

BOURASSA DC, MCMANUS IC, BRYDEN MP: Handedness and eye-dominance: a meta-analysis of their relationship. Laterality 1: 5-34, 1996.

BRADLEY MM, SAPIGAO RG, LANG PJ: Sympathetic ANS modulation of pupil diameter in emotional scene perception: effects of hedonic content, brightness, and contrast. Psychophysiol 54: 1419-1435, 2017.

BROWN JT, CONNELLY M, NICKOLS C, NEVILLE KA: Developmental changes of normal pupil size and reactivity in children. J Pediatr Ophthalmol Strabismus 52: 147-151, 2015.

BRUDER GE, STEWART JW, MCGRATH PJ: Right brain, left brain in depressive disorders: clinical and theoretical implications of behavioral, electrophysiological and neuroimaging findings. Neurosci Biobehav Rev 78: 178-191, 2017.

BURKHOUSE KL, OWENS M, FEURER C, SOSOO E, KUDINOVA A, GIBB BE: Increased neural and pupillary reactivity to emotional faces in adolescents with current and remitted major depressive disorder. Soc Cogn Affect Neurosci 12: 783-792, 2017

BURKHOUSE KL, SIEGLE GJ, GIBB BE: Pupillary reactivity to emotional stimuli in children of depressed and anxious mothers. J Child Psychol Psychiatry 55: 1009-1016, 2014.

BURTIS DB, KENNETH MH, MO J, WANG C, LEWIS GF, DAVILLA MI, DING M, PORGES SW, WILLIAMSON JB: The effects of constrained left versus right monocular viewing on the autonomic nervous system. Biol Psychol 100: 79-85, 2014.

COSTA VD, RUDEBECK PH: More than meets the eye: the relationship between pupil size and locus coeruleus activity. Neuron 89: 8-10, 2016. 
DALUWATTE C, MILES JH, CHRIST SE, BEVERSDORF DQ, TAKAHASHI TN, YAO G: Atypical pupillary light reflex and heart rate variability in children with autism spectrum disorder. J Autism Dev Disord 43: 1910-1925, 2013.

DAVIDSON RJ, TOMARKEN AJ: Laterality and emotion: an electrophysiological approach. In: Handbook of Neuropsychology. BOLTER F, GRAFMAN J (eds), Elsevier Science Publishers, Wisconsin, 1989, pp 419-441.

DISNER SG, BEEVERS CG, HAIGH EA, AARON TB: Neural mechanisms of the cognitive model of depression. Nat Rev Neurosci 12: 467-477, 2011.

DOS SANTOS JÚNIOR ED, DA SILVA AV, DA SILVA KR, HAEMMERLE CA, BATAGELLO DS, DA SILVA JM, LIMA LB, DA SILVA RJ, DINIZ GB, SITA LV, ELIAS CF, BITTENCOURT JC: The centrally projecting Edinger-Westphal nucleus--I: Efferents in the rat brain. J Chem Neuroanat 68: 22-38, 2015.

EBITZ RB, PLATT ML: Neuronal activity in primate dorsal anterior cingulate cortex signals task conflict and predicts adjustments in pupil-linked arousal. Neuron 85: 628-640, 2015.

GRAUR S, SIEGLE GJ: Pupillary motility: bringing neuroscience to the psychiatry clinic of the future. Curr Neurol Neurosci Rep 13: 365, 2013.

GRÜNEWALD BD, GREIMEL E, TRINKL M, BARTLING J, GROSSHEINRICH N, SCHULTE-KÖRNE G: Resting frontal EEG asymmetry patterns in adolescents with and without major depression. Biol Psychol 132: 212-216, 2018.

GÜNDOĞAN NU, YAZICI AC, SIMSEK A: Study on dominant eye measurement. Int J Ophthalmol 2: 271-277, 2009.

HALL CA, CHILCOTT RP: Eyeing up the future of the pupillary light reflex in neurodiagnostics. Diagnostics 8: 19, 2018.

HANKIN BL, GIBB BE, ABELA JR, FLORY K: Selective attention to affective stimuli and clinical depression among youths: role of anxiety and specificity of emotion. J Abnorm Psychol 119: 491-501, 2010.

HELLER W: Neuropsychological mechanisms of individual differences in emotion, personality, and arousal. Neuropsychol 7: 476-489, 1993.

HILZ MJ, DÜTSCH M, PERRINE K, NELSON PK, RAUHUT U, DEVINSKY O: Hemispheric influence on autonomic modulation and baroreflex sensitivity. Ann Neurol 49: 575-584, 2001.

JEFFERSON G: Substrates for integrative patterns in the reticular core. In: Reticular Formation of the Brain. SCHEIBEL ME, SCHEIBEL AB (eds), Little Brown, Boston, 1958, pp 31-68.

JOORMANN J, GOTLIB IH: Emotion regulation in depression: relation to cognitive inhibition. Cogn Emot 24: 281-298, 2010.

JOSHI S, LI Y, KALWANI RM, GOLD JI: Relationships between pupil diameter and neuronal activity in the locus coeruleus, colliculi, and cingulate cortex. Neuron 89: 221-324, 2016.

LI X, CHEN W, PAN K, LI H, PANG P, GUO Y, SHU S, CAI Y, PEI L, LIU D, AFEWERKY HK, TIAN Q, ZHU LQ, LU Y: Serotonin receptor 2c-expressing cells in the ventral CA1 control attention via innervation of the Edinger-Westphal nucleus. Nat Neurosci 21: 1239-1250, 2018.

LOBATO-RINCÓN LL, CABANILLAS-CAMPOS MD, BONNIN-ARIAS C, CHAMORRO-GUTIÉRREZ E, MURCIANO-CESPEDOSA A, SÁNCHEZ-RAMOS RODA C: Pupillary behavior in relation to wavelength and age. Front Hum Neurosci 8: 221, 2014.

MARCHEWKA A, ZURAWSKI Ł, JEDNORÓG K, GRABOWSKA A: The Nencki Affective Picture System (NAPS): introduction to a novel, standardized, wide-range, high-quality, realistic picture database. Behav Res Methods 46: 596-610, 2014.

MCDOUGAL DH, GAMLIN PD: Autonomic control of the eye. Compr Physiol 5: 439-473, 2015.

MESTANIK M, MESTANIKOVA A, VISNOVCOVA Z, CALKOVSKA A, TONHAJZEROVA I: Cardiovascular sympathetic arousal in response to different mental stressors. Physiol Res 64: 585-594, 2015.

MESTANIKOVA A, ONDREJKA I, MESTANIK M, CESNEKOVA D, VISNOVCOVA Z, BUJNAKOVA I, OPPA M, CALKOVSKA A, TONHAJZEROVA I: Pupillary light reflex is altered in adolescent depression. Physiol Res 66: 277-284, 2017. 
MESTANIKOVA A, MESTANIK M, ONDREJKA I, HRTANEK I, CESNEKOVA D, JURKO A, VISNOVCOVA Z, SEKANINOVA N, TONHAJZEROVA I: Complex cardiac vagal regulation to mental and physiological stress in adolescent major depression. $J$ Affect Disord 249: 234-241, 2019.

OTTAVIANI C, SHAPIRO D, DAVYDOV DM, GOLDSTEIN IB, MILLS PJ: The autonomic phenotype of rumination. Int J Psychophysiol 72: 267-275, 2009.

PEREIRA DM, KHAN A: Brain lateralization of emotional processing in depression. In: Depression. BREZNOSCAKOVA D (ed.), IntechOpen, Slovakia, 2017, pp 25-36.

PERRY VH, COWEY A: Retinal ganglion cells that project to the superior colliculus and pretectum in the macaque monkey. Neuroscience 12: 1125-1137, 1984.

PORGES SW: The polyvagal theory: new insights into adaptive reactions of the autonomic nervous system. Clev Clin J Med 76: 86-90, 2009.

PORGES SW, FURMAN SA: The early development of the autonomic nervous system provides a neural platform for social behaviour: a polyvagal perspective. Infant Child Dev 20: 106-118, 2011.

PROUDFOOT JG, JENKINS JL, BURGOON JK, NUNAMAKER JF: More than meets the eye: how oculometric behaviors evolve over the course of automated deception detection interactions. J Manag Inf Syst 33: 332-360, 2016.

SIEGLE GJ, STEINHAUER SR, CARTER CS, RAMEL W, THASE ME: Do the seconds turn into hours? Relationships between sustained pupil dilation in response to emotional information and self-reported rumination. Cognit Ther Res 27: 365-382, 2003.

SILK JS, STEINBERG L, MORRIS AS: Adolescents' emotion regulation in daily life: links to depressive symptoms and problem behavior. Child Dev 74: 1869-1880, 2003.

SILK JS, DAHL RE, RYAN ND, FORBES EE, AXELSON DA, BIRMAHER B, SIEGLE GJ: Pupillary reactivity to emotional information in child and adolescent depression: links to clinical and ecological measures. $\mathrm{Am}$ J Psychiatry 164: 1873-1880, 2007.

SILK JS, SIEGLE GJ, WHALEN DJ, OSTAPENKO LJ, LADOUCEUR CD, DAHL RE: Pubertal changes in emotional information processing: pupillary, behavioral, and subjective evidence during emotional word identification. Dev Psychopathol 21: 7-26, 2009.

STEINHAUER SR, HAKEREM G: The pupillary response in cognitive psychophysiology and schizophrenia. Ann N Y Acad Sci 658: 182-204, 1992.

STEVENS JS, HAMANN S: Sex differences in brain activation to emotional stimuli: a meta-analysis of neuroimaging studies. Neuropsychol 50: 1578-1593, 2012.

STONE LB, SILK JS, SIEGLE GJ, LEE KH, STROUD LR, NELSON EE, DAHL RE, JONES NP: Depressed adolescents' pupillary response to peer acceptance and rejection: the role of rumination. Child Psychiatry Hum Dev 47: 397-406, 2016.

THOMAS KM, DREVETS WC, DAHL RE, RYAN ND, BIRMAHER B, ECCARD CH, AXELSON D, WHALEN PJ, CASEY BJ: Amygdala response to fearful faces in anxious and depressed children. Arch Gen Psychiatry 58: 1057-1063, 2001.

TONHAJZEROVA I, JAVORKA K, PETRASKOVA M: Development of the heart rate variability parameter in young individuals aged 15-19 years. (Article in Slovak) Ces-Slov Pediat 54: 421-442, 1999.

TONHAJZEROVA I, JAVORKA K, JAVORKA M, PETRASKOVA M: Cardiovascular autonomic nervous system tests: reference values in young people (15-19 years) and influence of age and gender. Clin Physiol Funct Imaging 22: 398-403, 2002.

TONHAJZEROVA I, ONDREJKA I, JAVORKA K, TURIANIKOVA Z, FARSKY I, JAVORKA M: Cardiac autonomic regulation is impaired in girls with major depression. Prog Neuropsychopharmacol Biol Psychiatry 34: 613-618, 2010.

TONHAJZEROVA I, ONDREJKA I, CHLADEKOVA L, FARSKY I, VISNOVCOVA Z, CALKOVSKA A, JURKO A, JAVORKA M: Heart rate time irreversibility is impaired in adolescent major depression. Prog Neuropsychopharmacol Biol Psychiatry 39: 212-217, 2012.

TONHAJZEROVA I, MESTANIK M: New perspectives in the model of stress response. Physiol Res 66: 173-185, 2017. 
TRUEX RC, CARPENTER MB: Human Neuroanatomy. SUTIN J (ed.), Williams \& Wilkins, Baltimore, 1964, 591 p. WILHELM B, WILHELM H, LÜDTKE H: Pupillography: Principles and applications in basic and clinical research. In: Pupillography: Principles, Methods and Applications. KUHLMANN J, BOTTCHER M (eds), Zuckschwerdt Verlag, München, 1999, pp 1-11. 